\title{
CONOCIMIENTOS, ACTITUDES Y PRÁCTICAS DE LA MEDICINA BASADA EN EVIDENCIAS EN MÉDICOS ASISTENTES DE UN HOSPITAL PÚBLICO. LIMA- PERÚ
} Jorge Alfredo Segundo-Paredes ${ }^{1,5,8}$, Carlos Alejandro Gonzales-Medina $2,3,4,8$, Rosa Janeth Francia-De-la-Cruz
Edson Valdivia-Vera
5,7, Jacqueline Pamela Mejía-Veramendi ${ }^{8}$, Pedro M. Arango-Ochante

\begin{abstract}
RESUMEN
Objetivo. Determinar los conocimientos, actitudes y prácticas en Medicina Basada en Evidencias (MBE) en médicos asistentes de un hospital público. Materiales y métodos. Diseño analítico observacional de corte transversal, se utilizó un instrumento tipo cuestionario validado con un índice Kuder y Richarson (KR 20) de 0,79. Resultados. El conocimiento de la MBE es intermedio bajo en el $60 \%$ de los participantes. Además, existe una asociación estadística entre el número de horas dedicadas a la búsqueda de información y el nivel de conocimientos de MBE Conclusiones. Se requiere generar estrategias metodológicas de educación médica para mejorar el conocimiento de la MBE para optimizar las actitudes y prácticas de esta actividad medica
\end{abstract}

Palabras claves: Conocimientos; Actitudes; Prácticas; Medicina Basada en evidencias; Educación médica (Fuente: DeCS BIREME).

\section{KNOWLEDGE, ATTITUDES AND PRACTICES OF EVIDENCE-BASED MEDICINE IN ATTENDING PHYSICIANS OF A PUBLIC HOSPITAL. LIMA PERU}

\begin{abstract}
Objective. Determine the knowledge, attitudes and practices in Evidence Based Medicine (EBM) in attending physicians of a public hospital. Materials and methods. Analytical observational cross-sectional design, a validated instrument with a Kuder and Richarson index (KR 20) of 0,79 was used. Results. The knowledge of MBE is low intermediate in $60 \%$ of the participants. In addition, there is a statistical association between the number of hours dedicated to the search for information and the level of knowledge of MBE Conclusions. It is necessary to generate methodological strategies of medical education to improve the knowledge of the MBE to optimize the attitudes and practices of this medical activity
\end{abstract}

Keywords: Knowledge; Attitudes; Practices; Medicine Based on evidence; Medical education (Source: MeSH NLM).

\section{INTRODUCCIÓN}

La Medicina Basada en Evidencias (MBE) se constituye como un nuevo paradigma de la enseñanza y la práctica de la Medicina, la MBE promueve el uso de la mejor evidencia disponible, la experiencia clínica y las preferencias individuales del paciente. David Sackett acuño la definición de la MBE como el uso consciente, explícito y juicioso de las mejores y actuales pruebas en la toma de decisiones sobre el cuidado del paciente individual ${ }^{1}$.
Usar efectivamente la MBE requiere seguir un proceso adecuado para la toma de decisiones. Según recomienda el Centro de Medicina Basada en Evidencias de la Universidad de Oxford, se deben seguir 5 pasos: Paso 1 , formular correctamente la pregunta clínica, Paso 2, adquirir la mejor evidencia disponible, Paso 3, evaluar críticamente la evidencia, Paso 4, aplicar la evidencia en el paciente, y por último Paso 5, evaluar el impacto de la decisión tomada.

De todo el proceso anteriormente mencionado, la formulación de la pregunta clínica, constituye de vital

Médico Residente de Administración en Salud, Universidad Ricardo Palma, Dirección Regional de Salud del Callao

Médico Cirujano Especialista en Ginecología y Obstetricia Facultad de Medicina San Fernando, Universidad Nacional Mayor de San Marcos (UNMSM), Miembro de la Sociedad Peruana de Ginecología y Obstetricia SPOG, Perú

Fellow Junior ACOG, American College of Obstetricians and Gynecologists, United States of America

Unidad Funcional de Investigación del Instituto Materno Perinatal- Maternidad de Lima, Perú

Oficina de Gestión de Calidad. Hospital Nacional Madre Niño "San Bartolomé". Lima- Perú

Médico Especialista en Medicina de Rehabilitación. Hospital Nacional Daniel Alcides Carrión. Callao-Perú

Médico Especialista en Medicina Integral y Gestión en Salud, Universidad Nacional Mayor de San Marcos (UNMSM)

Asociación para el Desarrollo de la Investigación en Estudiantes de Ciencias de la Salud ADIECS, Lima-Perú

Citar como: Segundo-Paredes JA, Gonzales-Medina CA, Francia-De-La-Cruz RJ, Valdivia-Vera E, Mejía-Veramendi JP, Arango-Ochante PM . Conocimientos, actitudes y prácticas de la medicina basada en evidencias en médicos asistentes de un hospital público. Lima- Perú. Rev Peru Investig Matern Perinat 2018; 7(2): 9-15. https://doi.org/10.33421/inmp.2018112 
A

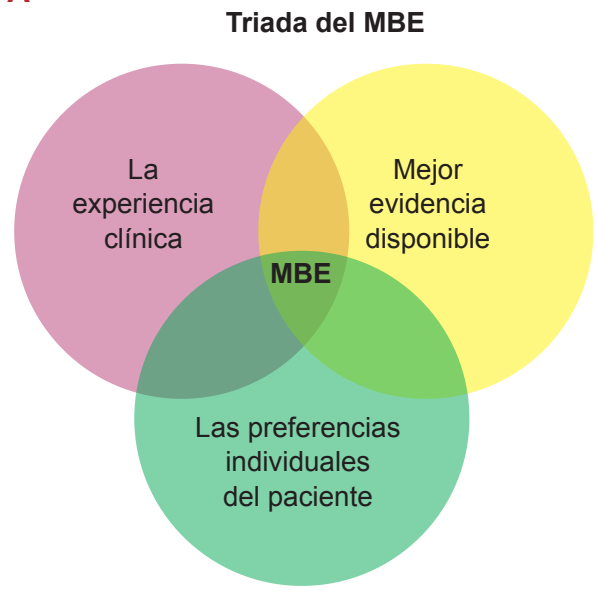

B

\section{El preceso de la MBE - Las 5 "A"}

Paso 4

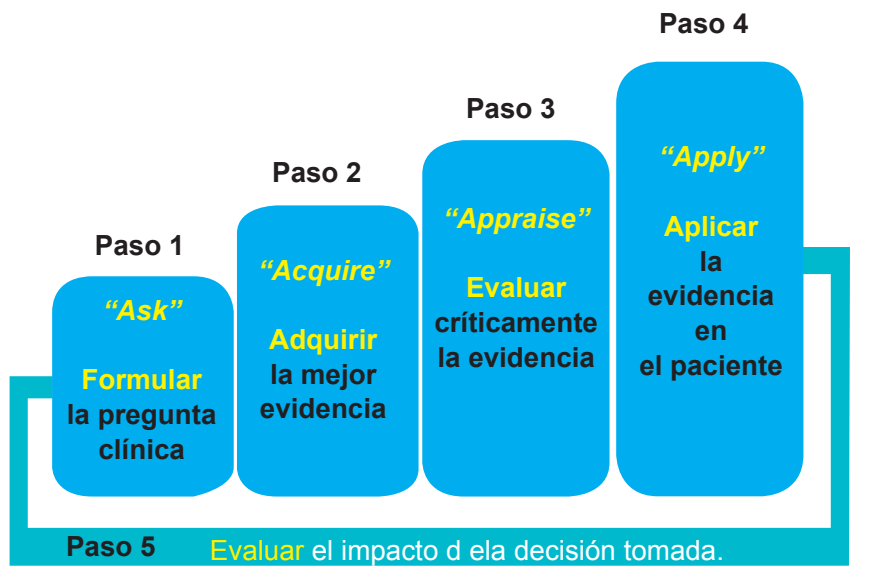

Figura 1. A) La triada de la Medicina Basada en evidencias. B) El proceso de la Medicina Basada en Evidencias las 5 "A". (Fuente: Traducido al español del Centro de Medicina Basada en Evidencias de la Universidad de Oxford)

importancia, es realizada en un formato estándar con el fin de facilitar la comunicación y entendimiento entre la comunidad científica y la médica en beneficio de los pacientes. Este formato estandarizado permite que la pregunta sea clara, precisa y enfocada para que la búsqueda y generación de evidencia sea lo más eficiente posible. Así, la estrategia de la pregunta "PICO" (por sus siglas en inglés, patient, intervention, comparison and outcome), incluye en su enunciado el tipo de Pacientes a los que se quiere beneficiar, la Intervención o tratamiento que se está analizando, el Comparador frente al que se evalúa dicha intervención y los Resultados (Outcome) que se esperan de dicha intervención y que se ajusten a las necesidades y valores de los paciente ${ }^{1}$.

En la práctica clínica diaria se necesita ofrecer a los pacientes la mejor evidencia disponible en relación al manejo, diagnostico, terapéutica y pronostico del daño a la salud. Es por ello que el ejercicio medico basado en MBE exige los conocimientos básicos y avanzados en lectura crítica y epidemiologia clínica. Esta última constituye una herramienta fundamental para entender la metodología de la investigación y la descripción de los indicadores epidemiológicos.

En el mundo, con la introducción de la MBE no se precisa el porcentaje de uso o al menos no hay un indicador para medir el uso correcto de la MBE en la práctica diaria. Sin embargo, mediante el advenimiento de la Auditoria Medica Basada en evidencias se puede conocer y medir el uso adecuado o no de la mejor evidencia disponible en la práctica diaria. De modo tal que se ofrece una atención medica de calidad.

La introducción de la MBE en la práctica diaria en nuestro medio no ha sido evaluada del todo; sin embargo, existen aproximaciones en relación a un grupo de profesionales de la salud sobre sus conocimientos, actitudes y prácticas ${ }^{3}$.

El objetivo primario del estudio fue determinar los conocimientos, actitudes y prácticas en MBE en un hospital público. Asimismo, como objetivos secundarios se evaluó la probable asociación entre los conocimientos, actitudes y prácticas en MBE frente a la edad, especialidad médica y tiempo de uso dedicado a la búsqueda de información en MBE.

\section{MATERIALES Y MÉTODOS}

El estudio fue observacional analítico de corte transversal mediante selección aleatoria de la población de médicos asistentes del Hospital Nacional Madre Niño "San Bartolomé" durante el periodo 2015 (criterio de elegibilidad). Se utilizó la fórmula de poblaciones finitas para el cálculo del tamaño muestral. A partir de un marco muestral correspondiente a 192 médicos asistentes con un error estándar del $5 \%$.

Se determinó el nivel de conocimientos actitudes y prácticas sobre Medicina Basada en Evidencias en médicos asistentes del Hospital Nacional Madre 
Niño "San Bartolomé" a través de un cuestionario autoaplicado que consta de dos secciones, la primera sobre datos socio epidemiológicos de los participantes (incluyendo edad, sexo, especialidad, año que realizo la residencia) y la segunda sección constó de 10 preguntas con opción múltiple sobre conocimientos de MBE. Las preguntas de actitudes y prácticas se elaboraron en escala de Likert y se incluyeron en el cuestionario final.

La validación del instrumento se realizó con un panel de expertos (un estadístico, un epidemiólogo y tres médicos con experiencia en la enseñanza del MBE). Se realizó el estudio piloto usando la metodología de validación de instrumentos con juicio de expertos que incluyó un Epidemiólogo Clínico, un Magister en Salud Publica, un Estadista en métodos cuantitativos y un experto en temas de Medicina Basada en Evidencias en la práctica de Ginecología y Obstetricia.

Además, se evaluó la confiabilidad por el método de testretest con el indicador Kappa. Se utilizó además el alfa de Cronbach para las estimaciones. Para comprobar la validez de los datos se realizó un análisis del patrón de respuesta general y de cada ítem (pruebas basadas en el proceso de respuesta), y un análisis factorial exploratorio de tipo componentes principales con rotación Varimax (prueba basada en la estructura interna). Se comprobó la pertinencia de la aplicación del análisis mediante el test de esfericidad de Bartlett (comprueba la hipótesis nula en que la matriz de correlaciones es la unidad) y el test de Kaiser-Meyer-Olkin (porcentaje de varianza compartida entre las variables).

Se usó el índice de Kappa estableciéndose una concordancia interobservador en relación a la congruencia interna del instrumento calificada como buena $(71,36 \%)$. Asimismo, se evaluó la confiabilidad con el índice Kuder y Richarson (KR 20) con un coeficiente de confiabilidad calificado como alto de 0,78 .

El análisis estadístico de los resultados se realizó en dos fases. La primera fase comprendió la estadística descriptiva en la que se analizó la frecuencia de los datos categóricos, según las características del estudio. Se analizó las variables cualitativas según prevalencias y proporciones. La segunda fase comprendió la estadística analítica en la que se exploró inicialmente la distribución de los datos para determinar la normalidad o no de los mismos utilizando adecuadamente pruebas no paramétricas o paramétricas, según fuese conveniente. Por ello, se realizó el estudio exploratorio mediante el uso de métodos gráficos (grafico $Q-Q$ plot y Box-plot) y

Tabla 1. Características generales de los participantes del estudio por especialidad médica

\begin{tabular}{|c|c|c|c|c|c|}
\hline & Gineco-Obstetricia & Pediatría & Cirugía Pediátrica & Otra & Total (N) \\
\hline \multicolumn{6}{|l|}{ Sexo } \\
\hline Masculino & $17,9 \%$ & $37,5 \%$ & $17,9 \%$ & $26,8 \%$ & 36 \\
\hline Femenino & $13,9 \%$ & $55,6 \%$ & $11,1 \%$ & $19,4 \%$ & 56 \\
\hline \multicolumn{6}{|l|}{ Estado civil } \\
\hline Casado & $8,6 \%$ & $43,0 \%$ & $20,7 \%$ & $27,6 \%$ & 58 \\
\hline Soltero & $36,6 \%$ & $33,3 \%$ & $23,8 \%$ & $23,8 \%$ & 21 \\
\hline Divorciado & $20,0 \%$ & $80,0 \%$ & $0 \%$ & $0 \%$ & 10 \\
\hline Conviviente & $0,1 \%$ & $33,3 \%$ & $33,3 \%$ & $33,3 \%$ & 3 \\
\hline \multicolumn{6}{|c|}{ Universidad de Origen } \\
\hline Publica & $36,6 \%$ & $33,3 \%$ & $23,8 \%$ & $23,8 \%$ & 61 \\
\hline Privada & $8,6 \%$ & $43,0 \%$ & $20,7 \%$ & $27,6 \%$ & 31 \\
\hline \multicolumn{6}{|c|}{$\begin{array}{l}\text { ¿Conoce la existencia de } \\
\text { cursos en MBE en el Perú? }\end{array}$} \\
\hline $\mathrm{Si}$ & $18,8 \%$ & $46,3 \%$ & $12,5 \%$ & $22,5 \%$ & 80 \\
\hline No & $0,1 \%$ & $33,3 \%$ & $33,3 \%$ & $33,3 \%$ & 12 \\
\hline \multicolumn{6}{|c|}{$\begin{array}{l}\text { ¿Ha participado en algún } \\
\text { curso de MBE? }\end{array}$} \\
\hline $\mathrm{Si}$ & $15,6 \%$ & $48,9 \%$ & $22,2 \%$ & $13,3 \%$ & 45 \\
\hline No & $17,0 \%$ & $40,4 \%$ & $8,5 \%$ & $34,0 \%$ & 47 \\
\hline
\end{tabular}


pruebas de contraste como Kolmogorov Smirnov para los datos mayores de 50 . Todo el análisis estadístico se realizó en el paquete estadístico SPSS versión 20.0. El nivel de significancia para todas las pruebas que se analizaron fue de un $p<0,05$.

\section{RESULTADOS}

La tasa de respuesta obtenida fue $83 \%$ y de los 92 médicos el rango de edad se hallaba entre 31 y 65 años con un promedio de 47 años (ver tabla 1). La mayor parte de los participantes eran casados $63 \%$ y el $65 \%$ eran egresados de universidades públicas.

La mayoría de los encuestados eran de la especialidad médica de pediatría (44,6\%). En la categoría otras especialidades se incluyeron con pocos participantes cirugía general, urología, medicina interna, cardiología y neurología. Estas especialidades tienen menor cantidad de especialista en la institución evaluada dado que es un hospital de atención materno infantil. El promedio de años de egresado de médico cirujano fue 20 años y el promedio de años de egresado de la especialidad fue 14 años (ver tabla 2). La nota promedio en la escala vigesimal fue 11,2 $\mathbf{4} \mathbf{4 , 7}$, calificando el nivel entre bajo e intermedio según lo señalado en la tabla 3 (figura 1).

Frente a un problema clínico el 50\% realiza una búsqueda bibliográfica por internet. Asimismo, el $20 \%$ se rige al protocolo o guías de la institución y el $16 \%$ consulta con un experto y/o colega. El $43 \%$ acude a dos bases de datos y solo el $8 \%$ accede a más de 5 bases de datos durante sus revisiones. Por otro lado, solo el $5 \%$ no accede a ninguna base de datos.

La cantidad de promedio de tiempo en horas/mes dedicada a la búsqueda de bibliografía biomédica en el $51,1 \%$ de los encuestados fue de 0 a 5 horas y solo el $10 \%$ dedicaba más de 20 horas mes en promedio como actividad académica.

El uso de PubMed y EMBASE se determinó en el $34 \%$ de los participantes. Ellos usan una hora semanal y solo el $4 \%$ de todos los participantes hacen uso de más de una hora semanal. Asimismo, el 34\% revisa un artículo semanal y el $9 \%$ un artículo diario. Cuando se interrogó sobre la periodicidad de la búsqueda de información el 18 $\%$ del total revisa un artículo mensual y el $26 \%$ un artículo quincenal.

En lo referente al conocimiento de términos técnicos utilizados en la MBE el $37 \%$ de los encuestados solo conoce y entiende los términos, el $13 \%$ conoce poco de los términos y solo el $2 \%$ desconoce los términos. El tipo de estudio que se prefiere revisar o leer frente a un problema clínico es la revisión sistemática.

Ante la pregunta si acude a cursos para desarrollar habilidades en la búsqueda de información por internet y la valoración crítica de la literatura biomédica el $65 \%$ respondió que acude ocasionalmente y el $15 \%$ lo hace frecuentemente. Sobre las revistas de especialidad que el medico asistente tiene acceso a texto completo

Tabla 2. Calificación de los niveles de conocimientos, actitudes y prácticas

\begin{tabular}{lcc}
\hline Variable & Calificación & Respuestas (si obtuvo una calificación) \\
\hline Conocimiento & Nivel alto & 16 a 20 \\
& Nivel intermedio & 11 a 15 \\
& Nivel bajo & 0 a 10 \\
Actitudes & Favorable & Respondió favorablemente a 6 o más preguntas sobre actitudes \\
& Desfavorable & Respondió desfavorablemente a menos de 6 preguntas sobre actitudes \\
Practicas & Indiferente & Respondió ni desfavorable ni favorablemente sobre actitudes \\
\hline & Correcta & Según la identificación correcta de un estudio clínico y su aplicación \\
\hline
\end{tabular}

Tabla 3. Características generales de los participantes del estudio por especialidad médica según tiempo de ejercicio profesional y nivel de conocimientos

\begin{tabular}{|c|c|c|c|c|c|}
\hline & Gineco-Obstetricia & Pediatría & Cirugía Pediátrica & Otra & Promedio \\
\hline \multicolumn{6}{|c|}{ Ejercicio profesional (Promedios) } \\
\hline Años de especialista & $13 \pm 2$ & $14 \pm 3$ & $15 \pm 2$ & $14 \pm 3$ & 14 años \\
\hline Años de médico cirujano & $19 \pm 3$ & $22 \pm 3$ & $21 \pm 3$ & $18 \pm 2$ & 20 años \\
\hline \multicolumn{6}{|l|}{ Conocimientos } \\
\hline Promedio (escala vigesimal) & 12,4 & 11,7 & 11,2 & 9,8 & 11.27 \\
\hline
\end{tabular}


Tabla 3. Distribución de frecuencias del total de encuestados según especialidad médica y los indicadores conocimientos actitudes y prácticas en Medicina Basada en Evidencias.

\begin{tabular}{|c|c|c|c|c|c|c|}
\hline & Ginecobstetricia & Pediatría & Cirugía Pediátrica & Otra * & Total & p-valor** \\
\hline \multicolumn{7}{|c|}{ Nivel de conocimientos } \\
\hline Alto & $20 \%$ & $1 \%$ & $29 \%$ & $50 \%$ & $10 \%$ & 0,002 \\
\hline Intermedio & $28 \%$ & $33 \%$ & $19 \%$ & $19 \%$ & $21 \%$ & 0,001 \\
\hline Bajo & $7 \%$ & $34 \%$ & $7 \%$ & $13 \%$ & $61 \%$ & 0,003 \\
\hline \multicolumn{7}{|l|}{ Actitudes } \\
\hline Favorable & $70 \%$ & $89 \%$ & $78 \%$ & $69 \%$ & $76 \%$ & 0,203 \\
\hline Indiferente & $6 \%$ & $7 \%$ & $8 \%$ & $6 \%$ & $7 \%$ & 0,102 \\
\hline Desfavorable & $15 \%$ & $18 \%$ & $17 \%$ & $17 \%$ & $17 \%$ & 0,123 \\
\hline \multicolumn{7}{|l|}{ Practicas } \\
\hline Incorrecta & $45 \%$ & $43 \%$ & $38 \%$ & $34 \%$ & $40 \%$ & 0,102 \\
\hline Correcta & $66 \%$ & $62 \%$ & $54 \%$ & $52 \%$ & $60 \%$ & 0,123 \\
\hline
\end{tabular}

* Otra especialidad médica incluyendo Cirugía General, Urología, Medicina Interna, Cardiología y Neurología. **Nivel de significancia de la prueba Chi Cuadrado de independenciaw

el $32 \%$ respondió que no tiene ningún acceso a su revista de la especialidad que es referente en su campo.

En cuanto a cómo se califica cada profesional sobre su habilidad para realizar literatura crítica sobre la literatura biomédica se evidencio que el $69 \%$ considera que posee una habilidad promedio, el $18 \%$ considera que su habilidad está por encima del promedio o es sobresaliente. Y solo el $11 \%$ refiere que su habilidad está por debajo o mínima del promedio.

El $80 \%$ de los encuestados refiere tener una predisposición favorable al aprendizaje basado en evidencias. El $80 \%$ considera que es favorable la mejor evidencia disponible para la contribución del razonamiento y aprendizaje clínico. Y el 84\% refiere una opinión favorable respecto a la utilidad de la mejor evidencia de investigación en la práctica clínica.

Con respecto a la búsqueda bibliográfica computarizada y al uso de base de datos informáticas para responder las preguntas de interés clínicos el $70 \%$ de los encuestados manifestó una actitud favorable. Asimismo, el $72 \%$ refiere una actitud desfavorable sobre la carencia de habilidades en la formulación de problemas y la valoración crítica de la literatura biomédica. Cuando se determinó la consistencia interna para medir la fiabilidad mediante el indicador de alfa de Cronbach se determinó un valor de 0,67 para la evaluación de la actitud en todas las categorías asignadas hallando que la actitud positiva hacia la MBE se obtuvo en el $82 \%$ de los encuestados.

El $70 \%$ refiere una predisposición favorable para colaborar con dedicación en la realización de investigación clínicamente relevante. El 60\% refiere una opinión desfavorable sobre la inasistencia a cursos de capacitación formal en estrategias efectivas para aprender y enseñar MBE. El 70\% considera que es desfavorable no introducir el aprendizaje basado en evidencia en el programa de segunda especialización. El $80 \%$ posee una opinión favorable para disponer de computadoras con servicio de internet las 24 horas del día y acceso libre a diferente base de datos en las sedes hospitalarias docentes del programa de segunda especialización.

Se determinó que existe una asociación estadística entre el nivel de conocimientos en MBE y el tipo de especialidad médica, no encontrándose dicha asociación numérica según el tipo de actitud y prácticas. Asimismo, el tipo de especialidad es independiente del nivel de conocimientos en MBE de forma global.

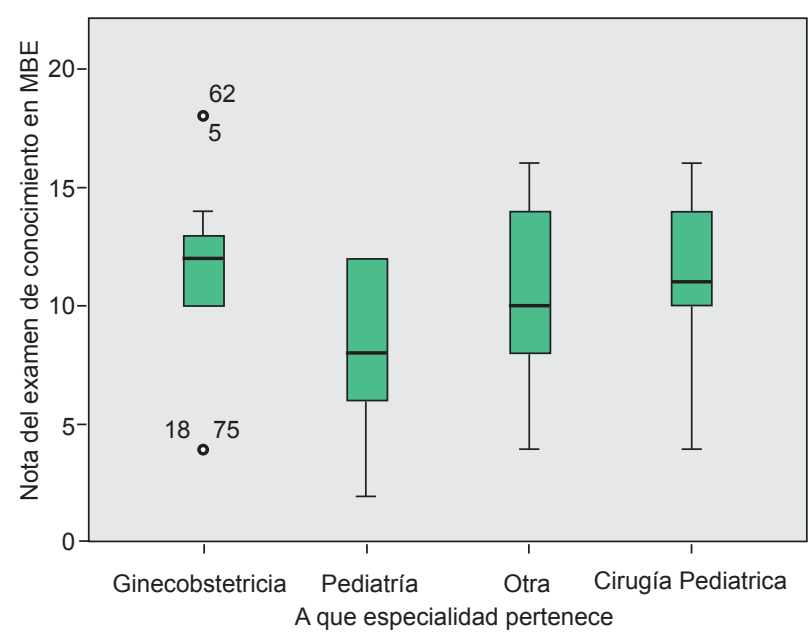

Figura 1. Diagrama Box Plot de la nota promedio obtenido en función de la especialidad médica sobre MBE. 


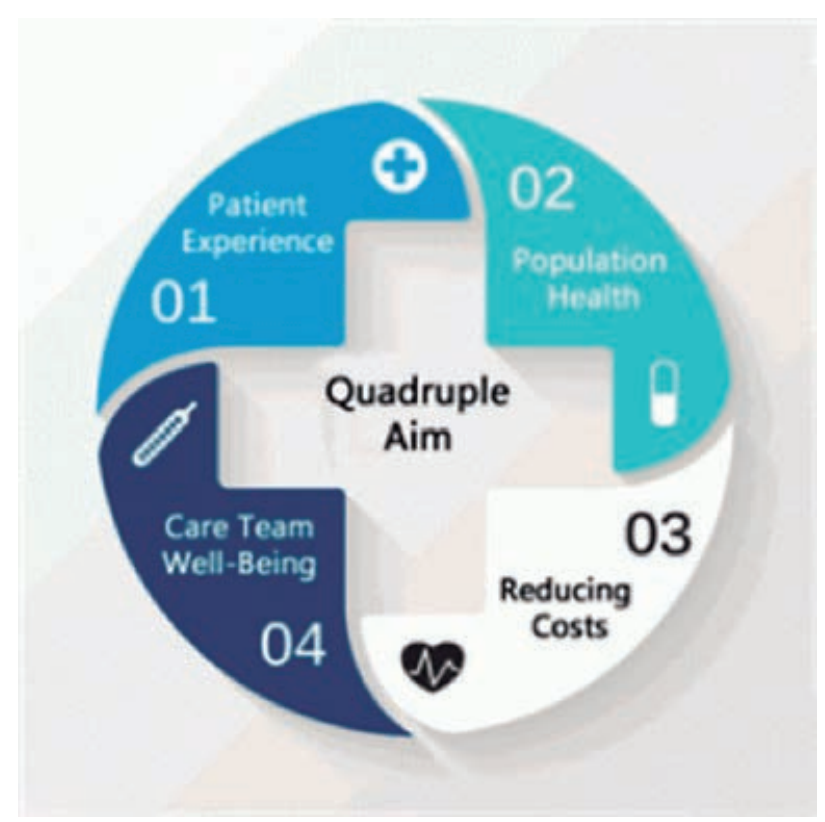

Fuente: Institute for Healthcare Improvement, Annals of Family Medicine, BMJ Quality \& Safety

\section{DISCUSIÓN}

Según el IHI (Institute for Healthcare Improvement), de los Estados Unidos plantea el Quadruple AIM "Cuádruple meta" mundial, para que todo sistema de salud sea sostenible en el tiempo. Dichas metas son: La experiencia del paciente, la salud de la población, reducir costos y finalmente el bienestar de los profesionales de salud.

La medicina basada en evidencias contribuye al cumplimiento de dichas metas, ya que fortalece la experiencia del paciente incluyéndolo en el proceso de toma de decisiones, lo que actualmente se denomina decisión compartida ("SDM: Share decisión making"). A la reducción de costos per capita, por el uso racional de los recursos en salud y a la salud de la población según el enfoque de salud pública basada en evidencias ${ }^{16}$.

Es conocido en la práctica médica el rol que cumplen los médicos asistentes en el manejo de situaciones clínicas y sobre todo de capacitación a los médicos residentes en formación para aprender y practicar objetivamente la Medicina Basada en Evidencias. Por ello es imprescindible la capacitación continua y actualizada.

La tasa de repuesta de la participación fue $83 \%$ como se verifica en diversos estudios en relación a este tema ${ }^{4,5}$.

Los médicos asistentes tienen una actitud favorable y adoptan como actividad necesaria el uso de la MBE en la práctica clínica como ocurre en diferentes publicaciones en las que las cifras son superiores al $50 \%$ de los encuestados. Por ejemplo en Australia $(82.7 \%)^{6}$ y Reino Unido (50.5\%) y en Arabia Saudita (60\%) en una encuesta a médicos asistenciales de atención hospitalaria y comunitaria ${ }^{7}$.

El valor de la evaluación de la actitud mediante el indicador alfa de Cronbach en nuestro estudio fue inferior al hallado en una investigación en Arabia Saudita, realizada en médicos asistentes, en los que se evaluó la actitud frente a las prácticas en MBE. En dicho estudio la tasa de actitud positiva hacia el MBE era $97 \%$ y con un porcentaje de uso del MBE en su práctica diaria de $48 \%$. Además, se reportó un nivel bajo de extracción de revisiones y bases de datos para el uso de la MBE. Se concluyó, por tanto, mejorar el entrenamiento en el conocimiento y búsqueda de información en sus participantes ${ }^{8}$.

Si bien en nuestro estudio el uso de la MBE por los médicos asistentes es aun limitado, es interesante cuando comparamos con los resultados de McAlister en el que el $33.1 \%$ de los participantes de su estudio siempre utilizan el MBE como herramienta y solo el $8.3 \%$ nunca lo han utilizado ${ }^{9}$.

La principal barrera identificada por los investigadores en relación a la práctica de la $\mathrm{MBE}$ es el tiempo disponible en la tercera parte de los entrevistados ${ }^{10}$ Sin embargo, se documentaba hace una década que la principal barrera era la falta de evidencia relevante en el $26 \%$ de los participantes. Esto último ha cambiado y se requieren estudios prospectivos para evaluarlo con objetividad.

Se documentó en el estudio de Arabia Saudita la necesidad de los médicos asistenciales en actualizarse en el manejo de las Guías de Práctica Clínicas (GPC). En ese contexto también se señaló como barrera para el uso de la MBE, la falta de capacitación en la unidades de posgrado universitarios, la inaccesibilidad por internet a la fuentes de información, la falta de practica en la interpenetración de los estudios clínicos ${ }^{11}$ y la falta de habilidades en búsqueda bibliográfica ${ }^{12}$.

En un estudio peruano realizado en dos hospitales peruanos se concluye que los médicos no tienen un uso adecuado de la MBE en su práctica clínica y que a principal barrera para la incorporación del MBE es la deficiente promoción de esta, se halló que no existían diferencias entre los médicos residentes encuestados y los médicos asistenciales ${ }^{13}$. Si bien esta investigación fue realizada hace diez años el grado de capacitación y las nuevas tecnologías de información aproximan más a los médicos asistenciales a mejorar su práctica de la MBE.

Un aspecto evaluado internacionalmente es la falta de capacitación en estadística médica, lo que impide llegar a una conclusión e interpretación correcta de los resultados ${ }^{13}$. En la investigación peruana comentada anteriormente se evaluó este aspecto haciendo participar a los encuestados con la redacción de la interpretación de cuatro términos básicos dela MBE, encontrando que solo el $17 \%$ fue capaz de definir correctamente dos de ellos ${ }^{3}$. 
Hay que considerar en un dato relevante en nuestro medio en una investigación publicada hace cuatro años en relación a los profesionales recién graduados. Se dice que existe una baja frecuencia de uso de fuentes de información como estrategia de búsqueda y se sugiere mayor capacitación dirigida a usar eficientemente los diversos recursos de información relacionados con salud. Esta debe ser de manera continua, en estudiantes de medicina y profesionales de la salud ${ }^{14}$. Además, se comenta que la falta del dominio del idioma ingles constituye un desafío adicional que limita la eficiente búsqueda bibliográfica. Este dato se estudió en médicos residentes mexicanos en formación de la especialidad de Ginecología y Obstetricia, encontrando deficiencias al respecto ${ }^{15}$.

En nuestra investigación no se halló diferencias estadísticas significativas entre los médicos asistentes de diversas especialidades médicas. Si bien hay un conocimiento aun inadecuado de la MBE la actitud hacia ella es muy favorable, sin embargo la práctica clínica aún sigue siendo incompleta. Además, reconocemos los probables sesgos de memoria que pueden ocurrir en los participantes.

Una de las limitaciones del estudio fue no evaluar con certeza el grado de interés de los participantes hacia el MBE y el análisis de los resultados comparativos sobre la práctica clínica asistencial en relación a estos temas en América Latina aun es limitado; y preponderantemente en la literatura mundial se publica estudios con mayor énfasis en los médicos de atención primaria.

Como conclusión final del estudio planteamos generar estrategias metodológicas de educación médica para mejorar el conocimiento de la MBE para optimizar las actitudes y prácticas de esta actividad médica.

Financiamiento: Autofinanciado.

Conflicto de interés: Los autores declaran no tener algún conflicto de intereses.

\section{REFERENCIAS BIBLIOGRÁFICAS}

1. Sacket D, Rosemberg W, Gray J, Haynes R, Richardson W. Evidence based medicine: what is and what it isn't. It's about integrating individual clinical expertise and the best external evidence. BMJ. 1996;312: 71-72.

2. Guyatt G, Meade MO, Richardson S, Jaeschke R. Chapter 3. What is the question? In: Guyatt G, Rennie D, Meade MO, Cook DJ, eds. Users' Guides to the Medical Literature:
A Manual for Evidence-Based Clinical Practice. 2nd ed. New York, NY: McGraw-Hill; 2008. http://www.jamaevidence. com/content/3348139.

3. Canelo C, Alarcon J, Amao E, Beteta V, Monge E. Conocimientos, actitudes y prácticas de la medicina basada en evidencias en médicos asistentes y residentes en dos hospitales de Lima-Perú. Rev Med Hered. 2007;18(2):7684.

4. Scott I, Heyworth R, Fairweather P. The use of evidencebased medicine in the practice of consultant physicians. Results of a questionnaire survey. Aust N Z J Med. 2000; 30: 319-326.

5. McColl A, Smith H, White P, Field J. General practitioner's perceptions of the route to evidence based medicine: a questionnaire survey. BMJ.1998; 316:361-365.

6. Veness M, Rikard-Bell G, Ward J. Views of Australian and New Zealand radiation oncologists and registrars about evidence-based medicine and their access to Internet based sources of evidence. Australas Radiol. 2003; 47: 409-415.

7. Al-Baghlie N, Al-Almaie SM. Physician attitudes towards evidence-based medicine in eastern Saudi Arabia. Ann Saudi Med. 2004; 24:25-428.

8. Al-Omari FK, Al-Asmary SM. Attitude, awareness and practice of evidence based medicine among consultant physicians in Western region of Saudi Arabia. Saudi Med J. 2006;27(12):1887-93

9. McAlister FA, Graham I, Karr GW, Laupacis A. EvidenceBased-Medicine and the Practicing Clinician. Journal of General Internal Medicine. 1999; 14: 236.

10. Igbal A, Glenny AM. General dental practitioners' knowledge of and attitude towards evidence based practice. Br Dent J. 2002; 193: 587-591.

11. Dawes M. On the need for evidence-based general and family practice. Evidence-Based Med. 1996; 1: 68-69.

12. Sabri AA, Qayyum MA. The problem of evidence-based medicine in developing countries. CMAJ. 2006;175: 62.

13. Evidence based Medicine Working Group. Evidence based medicine: a new approach to teaching the practice of medicine. JAMA.1992; 268 (17): 2420-2425.

14. Mejia CR, Caceres OJ, Vera CA, Nizama-Vía A, Curioso WH, Mayta-Tristán $P$, et al. Uso de fuentes de información en médicos recién graduados de Lima . Rev Peru Med Exp Salud Publica. 2014;31(4):716-20.

15. Veloz-Martínez MG, Almanza-Velasco E, Uribe-Ravell JA, González LL, Quintana-Romero V, Alanís-López P. Uso de tecnologías en información y comunicación por médicos residentes de ginecología y obstetricia. Inv Ed Med. 2012;1(4):183-9

16. EsSalud. Directiva No03-IETSI-ESSALUD-2016 "Normativa para la autorización y uso de productos farmacéuticos no incluidos en el petitorio farmacológico de EsSalud. Perú,2016.

\section{Correspondencia:}

Dr. Carlos Alejandro Gonzales Medina

Sector 2 Grupo 21 Manzana A Lote 24 Villa el Salvador

Email: cgonzales@iemp.gob.pe 